\title{
Direct EIT Jacobian calculations for conductivity change and electrode movement
}

\author{
Camille Gómez-Laberge and Andy Adler \\ Systems and Computer Engineering, Carleton University, Ottawa, Canada \\ E-mail: adler@sce.carleton.ca
}

\begin{abstract}
Electrical Impedance Tomography (EIT) is very sensitive to deformations of the medium boundary shape. For lung imaging, breathing and posture changes move the electrodes and change the chest shape, resulting in image artefacts. Several approaches have been proposed to improve the reconstructed images; most methods reconstruct both boundary deformation and conductivity change from the measured data. These techniques require calculation of the "movement Jacobian" reflecting measurement changes due to boundary deformation. Previous papers have calculated this Jacobian using perturbation techniques, which are slow (requiring multiple solutions of the forward problem) and become inaccurate with increasing finite element model size. This effect has limited reconstruction algorithms for deformable media to mostly $2 \mathrm{D}$.

To address this problem, we propose a direct method to calculate the Jacobian, based on a formulation of the derivatives of the finite element system matrix with respect to geometry changes. An illustrative example of these calculations is given as well as a comparison between the proposed method and a perturbation method. Results show this method is $\approx 300$ times faster; and for larger model sizes, the perturbation method begins to diverge from those from the direct method proposed.
\end{abstract}

Keywords: 3D Electrical Impedance Tomography, electrode movement, image reconstruction, inverse problem.

\section{Introduction}

In many clinical applications of EIT, the boundary shape changes during measurements. These boundary deformations and the associated electrode movements are known to cause significant image reconstruction artefacts. This effect is most severe in thoracic imaging. Lozano et al (1995) concluded that boundary deformations were primarily due to the expansion and contraction of the rib cage during breathing and postural changes occurring over long-term measurements. Adler and Guardo (1996) observed that boundary movement caused significant image artefacts, including broad central artefacts in the images.

In order to improve EIT images for media with deformable boundaries, several different algorithms have been proposed. The effects of electrode movement on 2D EIT images was studied by Blott et al (1998), who proposed a reconstruction matrix 
that minimises the measurement data sensitivity to movement by using a smoothing constraint. Later developments of 2D EIT imaging algorithms by Kolehmainen et al (2005) and Soleimani et al (2006) showed reconstructions of both conductivity change and electrode movement, where the latter group also showed results on a small 3D model. Lionheart (1998) showed that by extending the problem to 3D EIT, the measurement data are sufficient to determine both the conductivity distribution and the boundary shape; however, rotations, translations and scaling cannot be recovered.

To compensate for boundary deformation, all of the proposed methods require the calculation of a "movement Jacobian", which describes the change in measurements due to a deformation in the boundary. This matrix is calculated using perturbation techniques, by introducing small model deformations and repeatedly solving the forward problem. This has two disadvantages: it is slow, and it becomes inaccurate for large finite element models. For this reason, algorithms for deformable media EIT have been developed mainly for 2D problems.

To address this problem, this paper develops a direct method to calculate the Jacobian, based on a formulation of the derivatives of the finite element system matrix with respect to geometry changes. This paper expands on our recent work for developing a fast electrode movement Jacobian calculation (Gómez-Laberge and Adler, 2007) by first, formulating a mathematical model of the system geometry and its electrical parameters, and second, deriving the measurement sensitivity calculations used to directly calculate the Jacobian matrix for conductivity change and electrode movement. An illustrative example of these calculations is given as well as a comparison between the proposed method and an existing perturbation method using performance figures for the computation time and accuracy for calculations on four finite element models of varying complexity. The implementation of this method has been contributed to the EIDORS (Adler and Lionheart, 2006) version 3.2.

\section{Methods}

In this section, we develop an approach to calculate the Jacobian using a direct perturbation method for Finite Element Method (FEM) element conductivity changes and electrode movement. The modelling and formulation presented here extend the results of our previous work on electrode movement (Gómez-Laberge and Adler 2007). The EIT problem is formulated below for the 3D reconstruction of the conductivity distribution. Any differences for 2D modelling are indicated as required.

\subsection{The system model}

The system model considered here is a $3 \mathrm{D}$ FEM discretised into $n_{N}$ tetrahedral elements, which are formed from $n_{P}$ nodes. The conductivity distribution is constant on each element and is defined as the vector $\boldsymbol{\sigma} \in \mathbb{R}^{n_{N}}$. Along the boundary of the FEM are $n_{E}$ electrodes, each of which may be composed of a single node or by multiple nodes, as in 
the Complete Electrode Model (CEM). The potential difference between electrode $p$ and the reference is defined as $\phi_{p}$. While two electrodes stimulate the medium, the remaining electrodes measure the voltage between pairs. The $m^{\text {th }}$ measurement $v_{m}=\phi_{p}-\phi_{q}$, is the voltage between two electrodes $p$ and $q$. This stimulation process is repeated for each electrode pair; therefore, for $n_{E}$ electrodes, we obtain $n_{M}=n_{E}\left(n_{E}-3\right)$ voltage measurements $\mathbf{v} \in \mathbb{R}^{n_{M}}$.

The forward problem also depends on the position of every node $\mathbf{r} \in \mathbb{R}^{n_{D} \times n_{P}}$, where $n_{D}$ is the model dimension (e.g., $n_{D}=3$ for $\left.3 \mathrm{D}\right)$ and $\mathbf{r}_{p}=\left(r_{p x}, r_{p y}, r_{p z}\right)$ are the $(x, y, z)$ Cartesian coordinates of the nodes $1 \leq p \leq n_{P}$. In general, we will use $r_{p c}$ to refer to the coordinate $c$ of node $p$. We will model the movement of an electrode by displacing its representative node $\mathbf{r}_{p}$. For the case of the CEM, all constituent nodes of the electrode must be displaced. Hence, $\boldsymbol{\sigma}$ and $\mathbf{r}$ are used to construct the symmetric admittance matrix $\mathbf{Y} \in \mathbb{R}^{n_{P} \times n_{P}}$. This matrix associates each FEM element $k=1, \ldots, n_{N}$ to its conductivity $\sigma_{k}$ and its constituent nodes. The stimulation pattern used to inject current into the medium is represented by the matrix $\mathbf{Q} \in \mathbb{R}^{n_{P} \times n_{E}}$. Each column of $\mathbf{Q}$ indicates which electrodes are injecting current into the medium. The matrix product

$$
\mathbf{V}=\mathbf{Y}(\boldsymbol{\sigma}, \mathbf{r})^{-1} \mathbf{Q}
$$

yields the nodal potential difference matrix $\mathbf{V} \in \mathbb{R}^{n_{P} \times n_{E}}$, such that for stimulation $j=1, \ldots, n_{E}$, we have $\mathbf{V}_{p j}=\phi_{p}$ for nodes $p=1, \ldots, n_{P}$. Hence, the voltage measurement data $\mathbf{v}$ can be extracted from $\mathbf{V}$ by a series of element-wise subtractions (i.e., the measurement protocol), expressed using the operator $\mathbf{v}=T[\mathbf{V}]$. For instance, the $m^{\text {th }}$ measurement is obtained by $v_{m}=T[\mathbf{V}]_{m}=\mathbf{V}_{p j}-\mathbf{V}_{q j}$. The electrode resistance matrix $\mathbf{R} \in \mathbb{R}^{n_{E} \times n_{P}}$ given by

$$
\mathbf{R}=E\left[\mathbf{Y}(\boldsymbol{\sigma}, \mathbf{r})^{-1}\right]
$$

is also useful for sensitivity calculations. The $E$ operator simply extracts the rows from $\mathbf{Y}^{-1}$ corresponding to electrodes and also forces the reference node's potential to zero. For the CEM, the $E$ operator also extracts the complete electrode blocks augmenting the admittance matrix as defined by Polydorides and Lionheart (2002).

The admittance matrix from (1) can be written as a separable product

$$
\mathbf{Y}(\boldsymbol{\sigma}, \mathbf{r})=\mathbf{C}^{\top} \mathbf{S}(\mathbf{r}) \mathbf{D}(\boldsymbol{\sigma}) \mathbf{C}
$$

where $\mathbf{C} \in \mathbb{R}^{\left(n_{D}+1\right) n_{N} \times n_{P}}$ is the connectivity matrix, which associates each element to its vertices. The conductivity distribution is represented by $\mathbf{D}(\boldsymbol{\sigma}) \in \mathbb{R}^{\left(n_{D}+1\right) n_{N} \times\left(n_{D}+1\right) n_{N}}$ and is formed from

$$
\mathbf{D}(\boldsymbol{\sigma})=\operatorname{diag}(\boldsymbol{\sigma}) \otimes \mathbf{I}_{n_{D}+1},
$$

where $\otimes$ is the Kronecker product, and $\mathbf{I}_{n_{D}+1}$ is the $\left(n_{D}+1\right) \times\left(n_{D}+1\right)$ identity matrix. The matrix $\mathbf{S}(\mathbf{r}) \in \mathbb{R}^{\left(n_{D}+1\right) n_{N} \times\left(n_{D}+1\right) n_{N}}$ is called the unconnected system matrix, since it encapsulates the geometric properties of the FEM model. It is block-diagonal and 
defined as

Each block is given by

$$
\mathbf{S}=\left[\begin{array}{cccc}
\mathbf{S}_{1} & 0 & \cdots & 0 \\
0 & \mathbf{S}_{2} & & \vdots \\
\vdots & & \ddots & 0 \\
0 & \cdots & 0 & \mathbf{S}_{n_{N}}
\end{array}\right]
$$

$$
\mathbf{S}_{k}=\frac{2}{n_{D} !} \frac{1}{|\operatorname{det} \mathbf{A}|} \mathbf{B}^{\top} \mathbf{B} .
$$

The matrix $\mathbf{A}$ is calculated from the node positions of element $k$, which is shown for 3D and $2 \mathrm{D}$ respectively as

$$
\mathbf{A}=\left[\begin{array}{cccc}
1 & r_{x}^{1} & r_{y}^{1} & r_{z}^{1} \\
1 & r_{x}^{2} & r_{y}^{2} & r_{z}^{2} \\
1 & r_{x}^{3} & r_{y}^{3} & r_{z}^{3} \\
1 & r_{x}^{4} & r_{y}^{4} & r_{z}^{4}
\end{array}\right]^{-1}, \quad \mathbf{A}=\left[\begin{array}{rrr}
1 & r_{x}^{1} & r_{y}^{1} \\
1 & r_{x}^{2} & r_{y}^{2} \\
1 & r_{x}^{3} & r_{y}^{3}
\end{array}\right]^{-1}
$$

The matrix $\mathbf{B}$ is the submatrix of $\mathbf{A}$ with the top row deleted, denoted by $\mathbf{B}=[\mathbf{A}]_{\backslash \text { row1 }}$.

\subsection{Voltage measurement sensitivity calculations}

The Jacobian quantifies the sensitivity of the voltage measurements due to changes of the system model, in particular element conductivity changes and electrode movements. In this section, formulae for the voltage sensitivities $\partial \mathbf{V} / \partial \boldsymbol{\sigma}$ and $\partial \mathbf{V} / \partial \mathbf{r}$ are derived. In the following section, we describe how these quantities are assembled into each component of the Jacobian.

A change in the conductivity of an element $k$ requires the calculation of

$$
\frac{\partial \mathbf{V}}{\partial \sigma_{k}}=\frac{\partial}{\partial \sigma_{k}}\left(\mathbf{Y}^{-1} \mathbf{Q}\right)=\mathbf{Y}^{-1} \frac{\partial \mathbf{Y}}{\partial \sigma_{k}} \mathbf{Y}^{-\top} \mathbf{Q}=\mathbf{R} \frac{\partial \mathbf{Y}}{\partial \sigma_{k}} \mathbf{V},
$$

where, from (3), we obtain

$$
\frac{\partial \mathbf{Y}}{\partial \sigma_{k}}=\mathbf{C}^{\top} \mathbf{S} \frac{\partial \mathbf{D}}{\partial \sigma_{k}} \mathbf{C} .
$$

In order to evaluate (9) for a single element $k$, we need only consider the corresponding nodes and conductivity $\sigma_{k}$ of that element. Thus, we require the derivative

$$
\frac{\partial \mathbf{D}}{\partial \sigma_{k}}=\frac{\partial}{\partial \sigma_{k}}\left[\operatorname{diag}(\boldsymbol{\sigma}) \otimes \mathbf{I}_{n_{D}+1}\right]=\boldsymbol{\Xi}_{k} \otimes \mathbf{I}_{n_{D}+1}
$$

The matrix $\boldsymbol{\Xi}_{k} \in \mathbb{R}^{n_{N} \times n_{N}}$ is one on the $k^{\text {th }}$ position on the diagonal and zero elsewhere. Equation (10) models the change in conductivity of an element $k$. The conductivity perturbation is calculated by choosing the block $\mathbf{D}_{k}$ that modifies the conductivity of the nodes corresponding to this element.

Similar to (8), we consider the displacement of each node $p$ of an electrode along each coordinate $c$ independently. From (1),

$$
\frac{\partial \mathbf{V}}{\partial r_{p c}}=\mathbf{Y}^{-1} \frac{\partial \mathbf{Y}}{\partial r_{p c}} \mathbf{Y}^{-\top} \mathbf{Q}=\mathbf{R} \frac{\partial \mathbf{Y}}{\partial r_{p c}} \mathbf{V}
$$


where

$$
\frac{\partial \mathbf{Y}}{\partial r_{p c}}=\mathbf{C}^{\top} \frac{\partial \mathbf{S}}{\partial r_{p c}} \mathbf{D C}
$$

In order to evaluate (12), we need only consider the blocks $\mathbf{S}_{k}$ corresponding to elements in contact with the displaced electrode having the position vector $\mathbf{r}_{p}$. For each adjoining element $k$, we require the derivative

$$
\frac{\partial \mathbf{S}_{k}}{\partial \mathbf{r}_{p}}=\frac{\partial}{\partial \mathbf{r}_{p}}\left[\frac{2}{n_{D} !} \frac{1}{|\operatorname{det} \mathbf{A}|} \mathbf{B}^{\top} \mathbf{B}\right]
$$

Applying the product rule yields

$$
\frac{\partial \mathbf{S}_{k}}{\partial \mathbf{r}_{p}}=\frac{2}{n_{D} !}\left[\frac{\partial}{\partial \mathbf{r}_{p}}\left(\frac{1}{|\operatorname{det} \mathbf{A}|}\right) \mathbf{B}^{\top} \mathbf{B}+\frac{1}{|\operatorname{det} \mathbf{A}|}\left(\frac{\partial \mathbf{B}^{\top}}{\partial \mathbf{r}_{p}} \mathbf{B}+\mathbf{B}^{\top} \frac{\partial \mathbf{B}}{\partial \mathbf{r}_{p}}\right)\right] .
$$

However, the partial derivatives must be evaluated before (13) can be used. This can be done using a rank one matrix perturbation technique similar that shown in lemma 2 of Olsen and Gopinath (2004), as follows.

Rank one matrix perturbation: Let $\alpha \in \mathbb{R}, \mathbf{a}, \mathbf{b} \in \mathbb{R}^{d}$, and $\mathbf{X} \in \mathbb{R}^{d \times d}$ be an invertible matrix. Then, if $\alpha \neq-\left(\mathbf{b}^{\top} \mathbf{X} \mathbf{a}\right)^{-1}$, the rank one perturbed matrix $\mathbf{X}+\alpha \mathbf{a} \mathbf{b}^{\top}$ is invertible and

$$
\left(\mathbf{X}+\alpha \mathbf{a} \mathbf{b}^{\top}\right)^{-1}=\mathbf{X}^{-1}-\frac{\alpha \mathbf{X}^{-1} \mathbf{a b}^{\top} \mathbf{X}^{-1}}{1+\alpha \mathbf{b}^{\top} \mathbf{X}^{-1} \mathbf{a}} .
$$

In addition, the perturbation determinant is

$$
\operatorname{det}\left(\mathbf{X}+\alpha \mathbf{a} \mathbf{b}^{\top}\right)=\left(1+\alpha \mathbf{b}^{\top} \mathbf{X}^{-1} \mathbf{a}\right) \operatorname{det} \mathbf{X}
$$

Using the result in (14), the second term of (13) can be calculated for a perturbation of $\mathbf{r}_{p}$ along any direction in the limit $\alpha \downarrow 0$. Recall (7), and let $\mathbf{A}=\mathbf{P}^{-1}$. Then, for the corresponding blocks $\mathbf{S}_{k}$, we require the quantities

$$
\begin{aligned}
\frac{d \mathbf{B}}{d \alpha} & =\left.\frac{d \mathbf{A}}{d \alpha}\right|_{\backslash \text { row1 }} \\
& =\left.\frac{d}{d \alpha}\left[\left(\mathbf{P}+\alpha \mathbf{a} \mathbf{b}^{\top}\right)^{-1}\right]\right|_{\substack{\alpha=0 \\
\text { row1 }}} \\
& =\left[-\mathbf{P}^{-1} \mathbf{a} \mathbf{b}^{\top} \mathbf{P}^{-1}\right]_{\backslash \text { row1 }} \\
& =\left[-\mathbf{A} \mathbf{a} \mathbf{b}^{\top} \mathbf{A}\right]_{\backslash \text { row1 }}
\end{aligned}
$$

and similarly

$$
\frac{d \mathbf{B}^{\top}}{d \alpha}=\left(\frac{d \mathbf{B}}{d \alpha}\right)^{\top}=\left[-\mathbf{A}^{\top} \mathbf{b a}^{\top} \mathbf{A}^{\top}\right]_{\backslash \mathrm{col} 1} .
$$

The first term of (13) is calculated using (15):

$$
\begin{aligned}
\frac{d}{d \alpha} \frac{1}{|\operatorname{det} \mathbf{A}|} & =\frac{-\operatorname{sgn}(\operatorname{det} \mathbf{A})}{\operatorname{det} \mathbf{A}^{2}} \frac{d}{d \alpha} \operatorname{det} \mathbf{A} \\
& =\left.\frac{-\operatorname{sgn}(\operatorname{det} \mathbf{A})}{\operatorname{det} \mathbf{A}^{2}} \frac{d}{d \alpha} \operatorname{det}\left[\left(\mathbf{P}^{-1}+\alpha \mathbf{a} \mathbf{b}^{\top}\right)^{-1}\right]\right|_{\alpha=0} \\
& =\frac{1}{|\operatorname{det} \mathbf{A}|} \mathbf{b}^{\top} \mathbf{A} \mathbf{a}
\end{aligned}
$$


where the $\operatorname{sign}$ function $\operatorname{sgn}(x)$ returns the sign of a real number

$$
\operatorname{sgn}(x)= \begin{cases}-1 & \text { if } x<0 \\ 0 & \text { if } x=0 \\ 1 & \text { if } x>0\end{cases}
$$

Substituting (16) and (17) into (13) yields the sensitivity term for each element adjoining the electrode represented by $\mathbf{r}_{p}$

$$
\frac{\partial \mathbf{S}_{k}}{\partial r_{p c}}=\frac{2}{n_{D} !}\left[\frac{1}{|\operatorname{det} \mathbf{A}|}\left(\mathbf{b}^{\top} \mathbf{A} \mathbf{a} \mathbf{B}^{\top} \mathbf{B}+\frac{\partial \mathbf{B}^{\top}}{\partial r_{p c}} \mathbf{B}+\mathbf{B}^{\top} \frac{\partial \mathbf{B}}{\partial r_{p c}}\right)\right] .
$$

Equation (18) is the explicit form of (13) and models the deformation of one element adjoining the displaced electrode with position vector $\mathbf{r}_{p}$. This equation is also a discrete form of the Lie derivative of the conductivity tensor (Lionheart, 2004). For each deformed element, the perturbations along $x, y$, and $z$ are calculated by choosing the unit vectors $\mathbf{a}$ and $\mathbf{b}$ in (16) and (17) that modify the coordinates of the corresponding node. These coordinate data are stored as in equation (7). Thus, the FEM system matrix $\mathbf{S}$ is constructed as in equation (5), where only the element blocks $\mathbf{S}_{k}$ adjoining the displaced electrode are non-zero. Let $N(p)$ be the set of elements which contain the node $p$. Then, the full matrix, of same size as $\mathbf{S}$, can be represented by the sum

$$
\frac{\partial \mathbf{S}}{\partial r_{p c}}=\sum_{k \in N(p)} \boldsymbol{\Xi}_{k} \otimes \frac{\partial \mathbf{S}_{k}}{\partial r_{p c}}
$$

\subsection{A direct calculation of the Jacobian matrix}

Given the measurement sensitivities from (10) and (18), we calculate a component strictly due to changes in element conductivity and another strictly due to electrode movements. Together these form the augmented Jacobian matrix

$$
\mathbf{J}=\left[\mathbf{J}_{c} \mathbf{J}_{m}\right] \in \mathbb{R}^{n_{M} \times\left(n_{N}+n_{D} n_{E}\right)} .
$$

Both components of the Jacobian are formed in the same way. In each case, a subcomponent for each stimulation pattern $1 \leq j \leq n_{E}$ is assembled as follows

$$
\mathbf{J}_{c}=\left[\begin{array}{c}
\mathbf{J}_{c 1} \\
\vdots \\
\mathbf{J}_{c n_{E}}
\end{array}\right], \mathbf{J}_{m}=\left[\begin{array}{c}
\mathbf{J}_{m 1} \\
\vdots \\
\mathbf{J}_{m n_{E}}
\end{array}\right], \mathbf{J}_{c j} \in \mathbb{R}^{\left(n_{E}-3\right) \times n_{N}}, \mathbf{J}_{m j} \in \mathbb{R}^{\left(n_{E}-3\right) \times n_{D} n_{E}} .
$$

The sensitivity matrices for the $j^{\text {th }}$ stimulation pattern are formed using column $j$ of (8) and (11) for all elements $k=1, \ldots, n_{N}$. These are denoted by $\partial \mathbf{V}_{j} / \partial \sigma_{k}$ and $\partial \mathbf{V}_{j} / \partial \mathbf{r}_{p}$ and contribute to the subcomponents

$$
\begin{aligned}
& \mathbf{J}_{c j}=M\left[\frac{\partial \mathbf{V}_{j}}{\partial \sigma_{1}} \cdots \frac{\partial \mathbf{V}_{j}}{\partial \sigma_{n_{N}}}\right], \\
& \mathbf{J}_{m j}=M\left[\frac{\partial \mathbf{V}_{j}}{\partial r_{1 x}} \frac{\partial \mathbf{V}_{j}}{\partial r_{1 y}} \frac{\partial \mathbf{V}_{j}}{\partial r_{1 z}} \cdots \frac{\partial \mathbf{V}_{j}}{\partial r_{n_{E}}} \frac{\partial \mathbf{V}_{j}}{\partial r_{n_{E} y}} \frac{\partial \mathbf{V}_{j}}{\partial r_{n_{E} z}}\right] .
\end{aligned}
$$

Similar to $T$, the operator $M$ extracts the measurements taken from a pair of available electrodes during stimulation $j$ according to order specified in the measurement protocol. 


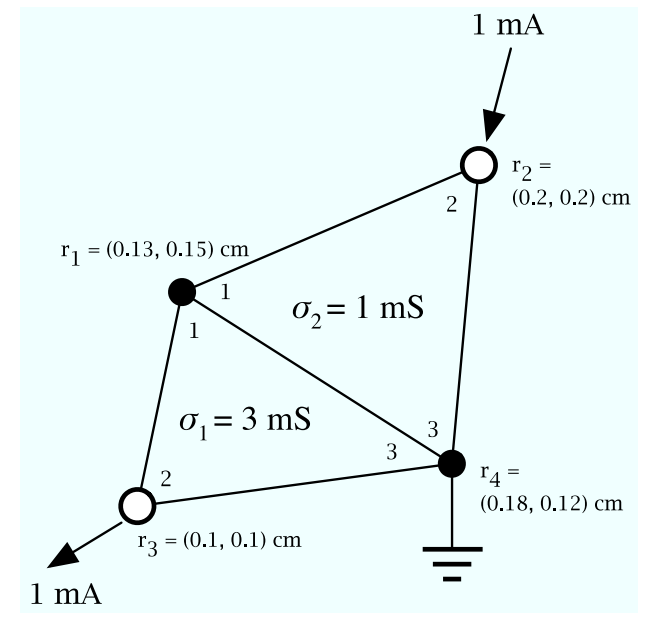

Figure 1. Illustrative example of a two-element FEM with two electrodes (open circles) and four nodes.

\subsection{An illustrative example}

Figure 1 shows a simple, two-element FEM. The organisation of the nodes and elements, however, is equivalent to the ones used for typical EIT FEM. For example, element 1 has conductivity $\sigma_{1}=3 \mathrm{mS}$ and corresponds with nodes $\mathbf{r}_{1}, \mathbf{r}_{3}$ and $\mathbf{r}_{4}$. Observe that the element also has an internal labelling of these nodes: 1, 2 and 3, respectively. The two electrodes, shown as open circles, are labelled as nodes $\mathbf{r}_{2}$ and $\mathbf{r}_{3}$; they stimulate the circuit with $1 \mathrm{~mA}$ of current. The reference node is $\mathbf{r}_{4}$, whence all node potentials and resistances are measured. The system model as described in section 2.1 is

$$
\begin{aligned}
& \mathbf{C}=\left[\begin{array}{llll}
1 & 0 & 0 & 0 \\
0 & 0 & 1 & 0 \\
0 & 0 & 0 & 1 \\
1 & 0 & 0 & 0 \\
0 & 1 & 0 & 0 \\
0 & 0 & 0 & 1
\end{array}\right], \quad \mathbf{D}=\left[\begin{array}{llllll}
3 & 0 & 0 & 0 & 0 & 0 \\
0 & 3 & 0 & 0 & 0 & 0 \\
0 & 0 & 3 & 0 & 0 & 0 \\
0 & 0 & 0 & 1 & 0 & 0 \\
0 & 0 & 0 & 0 & 1 & 0 \\
0 & 0 & 0 & 0 & 0 & 1
\end{array}\right] \mathrm{mS}, \\
& \mathbf{S}=\left[\begin{array}{ccccccc}
2 & -1 & -1 & 0 & 0 & 0 \\
-1 & 1 & 0 & 0 & 0 & 0 \\
-1 & 0 & 1 & 0 & 0 & 0 \\
0 & 0 & 0 & 1.48 & -0.30 & -1.17 \\
0 & 0 & 0 & -0.30 & 0.74 & -0.43 \\
0 & 0 & 0 & -1.17 & -0.43 & 1.61
\end{array}\right], \quad \mathbf{Q}=\left[\begin{array}{c}
0 \\
1 \\
-1 \\
0
\end{array}\right] \mathrm{mA} .
\end{aligned}
$$

The connectivity matrix $\mathbf{C}$ indicates the correspondence between the global node labels $\mathbf{r}_{k}$ and the internal node labels for each element. For example, from figure 1 the internal label for node 3 of element 1 corresponds to $\mathbf{r}_{4}$; hence, row 3 , column 4 of $\mathbf{C}$ is set to one. The conductivity matrix $\mathbf{D}$ is diagonal, where all nodes corresponding to the same element have the value $\sigma_{k}$. The FEM system matrix is block-diagonal as in (5). The 
block $\mathbf{S}_{1}$, for example, is formed using the geometry data as in (6) and (7)

$$
\mathbf{A}=\left[\begin{array}{ccc}
1 & 0.13 & 0.15 \\
1 & 0.1 & 0.1 \\
1 & 0.18 & 0.12
\end{array}\right]^{-1} \quad \mathrm{~cm}^{-1} \Rightarrow \mathbf{B}=\left[\begin{array}{ccc}
-588 & -882 & 1471 \\
2353 & -1471 & -882
\end{array}\right] \mathrm{cm}^{-1}
$$

The voltages and resistances are calculated as in (1) and (2), where the fourth row and column are held at zero, since node $\mathbf{r}_{4}$ is reference. These equations give

$$
\mathbf{V}=\left[\begin{array}{c}
-0.14 \\
1.30 \\
-0.47 \\
0
\end{array}\right] \mathrm{V}, \quad \mathbf{R}=\left[\begin{array}{cccc}
94.60 & 1392.00 & 94.60 & 0 \\
230.00 & 94.60 & 563.10 & 0
\end{array}\right] \Omega
$$

For this example, we calculate the voltage measurement sensitivities for a change in conductivity in $\sigma_{1}$ and a displacement of electrode $\mathbf{r}_{2}$. From (10) and (4), only the first three rows and columns survive the differentiation. Thus, according to (8), we obtain

$$
\frac{\partial \mathbf{V}}{\partial \sigma_{1}}=\mathbf{R C}^{\top} \mathbf{S} \frac{\partial \mathbf{D}}{\partial \sigma_{1}} \mathbf{C V}=\left[\begin{array}{c}
-12.78 \\
-142.16
\end{array}\right] \mathrm{V} \cdot \mathrm{mS}^{-1}
$$

To calculate the sensitivity due to a displacement of $\mathbf{r}_{2}$, only the last three rows and columns do not vanish. For a displacement in the $y$ direction, we must perturb element $r_{y}^{2}$ in $\mathbf{A}$ from $\mathbf{S}_{2}$. Thus, the rank one perturbation vectors are $\mathbf{a}=\left[\begin{array}{lll}0 & 1 & 0\end{array}\right]^{\top}$ and $\mathbf{b}=\left[\begin{array}{lll}0 & 0 & 1\end{array}\right]^{\top}$. To evaluate (18), we compute

$$
\begin{aligned}
\frac{\partial \mathbf{B}}{\partial r_{2 y}}= & {\left[\begin{array}{ccc}
-0.28 & -0.71 & 0.99 \\
-0.47 & -1.18 & 1.65
\end{array}\right] } \\
\frac{\partial \mathbf{S}_{2}}{\partial r_{2 y}}= & {\left[\begin{array}{ccc}
1871.50 & -321.40 & -1550.10 \\
-321.40 & -808.40 & 1124.80 \\
-1550.10 & 1124.80 & 425.30
\end{array}\right] \times 10^{6} . }
\end{aligned}
$$

The required sensitivity obtained from (11) is

$$
\frac{\partial \mathbf{V}}{\partial r_{2 y}}=\left[\begin{array}{c}
-14.54 \\
-2.48
\end{array}\right] \mathrm{V} \cdot \mathrm{cm}^{-1}
$$

All other sensitivity calculations are done in this way. Once these are complete, the Jacobian components are assembled directly from these matrices as shown in (20).

\section{Results}

In this section, we describe the performance of the proposed method on several 3D FEM of distinct element density. The FEM used in this paper are constructed by extruding a 2D circular FEM into a 3D cylinder with tetrahedral elements. Sixteen electrodes are placed around the cylindrical circumference in correspondence with a typical experimental electrode configuration. Point electrodes are considered, which 


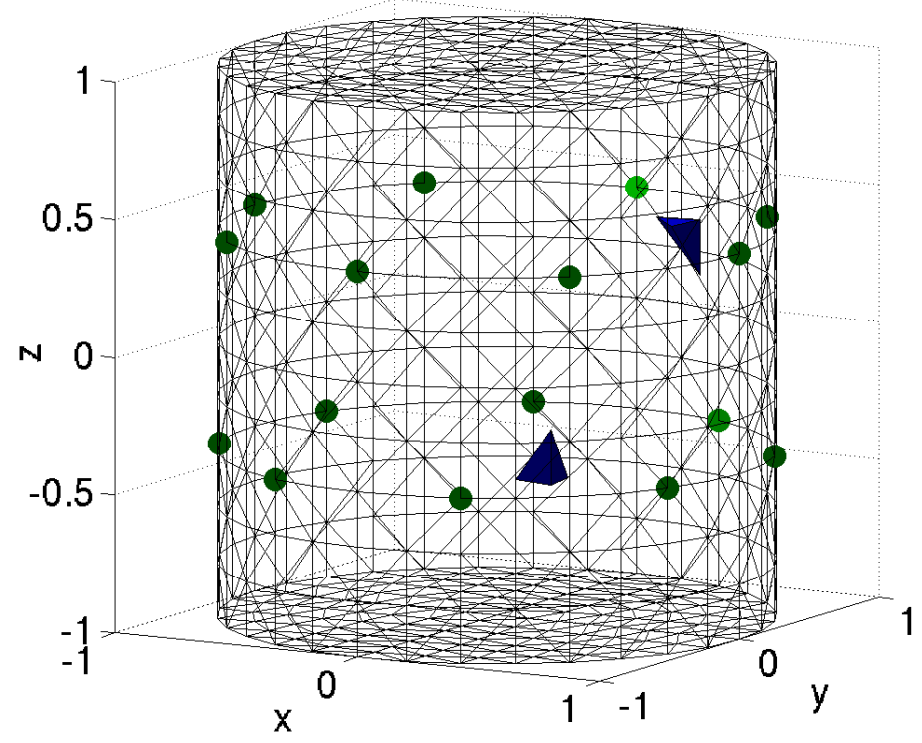

Figure 2. A forward model FEM with 7,680 tetrahedral elements and 1,595 nodes. The 16 point electrodes are marked by green discs. Two contrast elements are shown in blue.

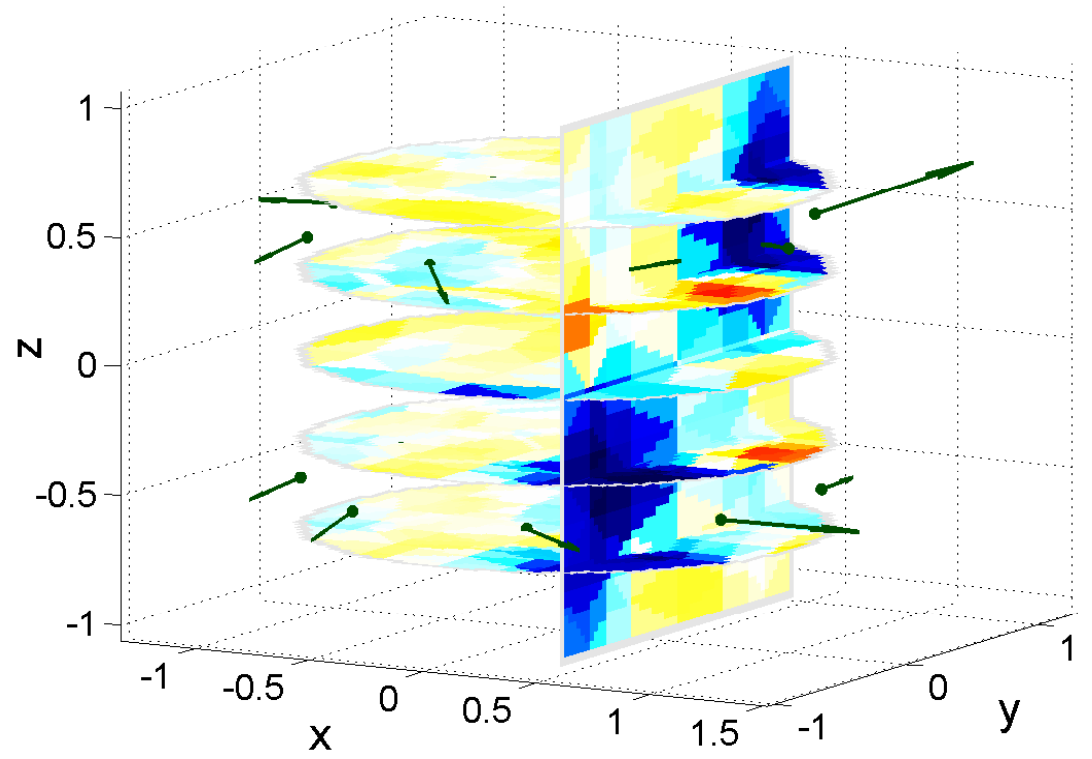

Figure 3. Reconstruction of the FEM in figure 2 using the direct method on a coarser FEM with 1,536 elements and 697 nodes. The indirect method produces a virtually identical result. Electrode positions and reconstructed displacements are shown as the small green discs and arrows, respectively.

are directly connected to the nodes on the model boundary. Figure 2 illustrates a 7,680-element FEM model with the electrode configuration used in this paper. The 
Table 1. FEM Forward and Inverse Model Pairs

\begin{tabular}{|c||r|r|r|r|}
\hline \multicolumn{1}{|c||}{$\begin{array}{c}\text { Model } \\
\text { pair }\end{array}$} & \multicolumn{2}{c|}{ Elements } & \multicolumn{2}{c|}{ Nodes } \\
\cline { 2 - 5 } & Forward & Inverse & Forward & Inverse \\
\hline$A$ & 7,680 & 1,536 & 1,595 & 369 \\
$B$ & 15,360 & 3,072 & 3,045 & 697 \\
$C$ & 30,720 & 5,995 & 6,144 & 1,305 \\
$D$ & 61,440 & 11,445 & 12,288 & 2,465 \\
\hline
\end{tabular}

Table 2. Jacobian Calculation Time and Relative Error

\begin{tabular}{|c||r|r|c|}
\hline \multicolumn{1}{|c||}{$\begin{array}{c}\text { Model } \\
\text { pair }\end{array}$} & \multicolumn{2}{|c|}{ Computation Time (s) } & \multicolumn{1}{c|}{ Relative Error } \\
\cline { 2 - 4 } & Direct & Indirect & $\left\|\mathbf{J}_{\text {indir }}-\mathbf{J}_{\text {dir }}\right\|_{F} /\left\|\mathbf{J}_{\text {dir }}\right\|_{F}$ \\
\hline$A$ & 2.96 & 621.68 & $9.33 \times 10^{-7}$ \\
$B$ & 6.56 & $1,665.50$ & $9.93 \times 10^{-7}$ \\
$C$ & 18.93 & $5,531.40$ & $1.22 \times 10^{-6}$ \\
$D$ & 85.26 & $11,685.00$ & $1.28 \times 10^{-6}$ \\
\hline
\end{tabular}

electrodes are numbered in a "zig-zag" pattern described by Graham and Adler (2007), where electrode 1 (light), electrode 2 (medium), and the remaining electrodes (dark) are shown by green discs. The two blue elements shown in this figure are contrast elements used to compare image reconstructions. These contrasts have a conductivity of 1.5 times that of the background.

The computations of the conductivity distribution depend on the quantities derived from the FEM geometry. Hence, we consider four model densities, successively differing in number of elements by a factor of 2 . Table 1 summarises the four FEM model pairs used, labelled $A$ through $D$.

Image reconstructions were computed using the proposed method and were compared with the previously used indirect perturbation method described by Soleimani et al (2006). The indirect perturbation method requires that the forward problem be solved repeatedly, after small changes for each element conductivity and electrode position. Moreover, the magnitude of these perturbations must be carefully chosen, i.e., small enough to accurately represent the system sensitivity yet avoid finite-precision error.

Table 2 shows the average computation time required in seconds to compute the Jacobian matrix for each FEM model and the relative error between both methods using the Frobenius norm. Calculations were performed on an IBM Intellistation A Pro equipped with 64-bit AMD Opteron CPU running at 2.0 GHz with $8 \mathrm{~GB}$ RAM. The software was implemented and executed using MATLAB v.7 (2006a) under the Electrical Impedance and Diffuse Optical Tomography (EIDORS) suite, v.3.2 (Adler and Lionheart 2006). For FEM with fewer elements than model pair $A$ the performance results between both methods are comparable, since relatively few forward solutions 
need to be computed in the indirect method. For large FEM, the indirect method requires a significantly longer computation time and memory.

Figure 3 shows the image reconstruction obtained where the Jacobian was calculated with the direct method; the indirect method produced a virtually identical image. Reconstructions were computed in EIDORS (Adler and Lionheart, 2006) version 3.2 using the regularised linear inverse solution with hyperparameters $\lambda=3 \times 10^{-3}$ and $\mu=20$. The electrodes were slightly displaced (less than $1 \%$ of the model diameter) in simulations using the FEM model shown in figure 2. Five horizontal slices at $z=0, \pm 0.35, \pm 0.70$ are shown where the electrode positions are indicated by the small green circles; their reconstructed displacements are represented by the green arrows. One vertical slice cuts through the reconstructed contrasts along $x=0.5$. The position of both contrast elements in blue has been recovered.

\section{Discussion}

In this study, we formulate a more efficient and accurate computation of the electrode movement Jacobian by eliminating the need of re-calculation of the forward problem and the numerical error associated with numerical perturbations. This was accomplished by implementing a rank one perturbation technique similar to that described by Olsen and Gopinath (2004), directly on the FEM system matrix. The method developed here applies to a general FEM geometry in 2 or 3D equipped with either single- or multiplenode electrodes. The CEM as formulated by Polydorides and Lionheart (2002) may also apply the method after having considered extensions for the augmented complete electrode blocks in the full system matrix.

Kaipio et al (2000) formulate a direct calculation for the conductivity Jacobian akin to ours. Polydorides and Lionheart (2002) derive an adjoint field method to calculate the conductivity Jacobian and claim significant speed improvements. However, we are not aware of any other calculations for the movement Jacobian except for the indirect perturbation method used by Soleimani et al (2006).

The performance results are significantly faster than the previous, indirect perturbation method and maintains good numerical agreement. The reconstructed images are similar to the indirect method and show no significant change in

reconstruction fidelity. Such a calculation method may be useful in reconstruction algorithm design for large FEM inverse problems.

\section{Acknowledgments}

This work was supported by a grant from the Natural Sciences and Engineering Research Council of Canada. 


\section{References}

Adler A and Guardo R 1996 Electrical impedance tomography: regularized imaging and contrast detection IEEE Trans. Med. Imag. 15 170-179

Adler A and Lionheart W R B 2006 Uses and abuses of EIDORS: and extensible software base for EIT Physiol. Meas. 27 S25-S42

Blott B H, Daniell G J and Meeson S 1998 Electrical impedance tomography with compensation for electrode positioning variations Phys. Med. Biol. 43 1731-1739

Graham B M and Adler A 2007 Electrode placement strategies for 3D EIT Physiol. Meas. 28 S29-S44

Gómez-Laberge C and Adler A 2007 Direct calculation of the electrode movement Jacobian for 3D EIT IFMBE Proc. XIII Int. Conf. Elec. Bioimped. \&3 VIII Conf. Elec. Imped. Tomog. 17 364-367

Kaipio J P, Kolehmainen V, Somersalo E and Vauhkonen M 2000 Statistical inversion and Monte Carlo sampling methods in electrical impedance tomography Inverse Problems 16 1487-1522

Kolehmainen V, Lassas M and Ola P 2005 The inverse conductivity problem with an imperfectly known boundary SIAM J. Appl. Math. 66 365-383

Lionheart W R B 1998 Boundary shape and electrical impedance tomography Inverse Problems 14 139-147

Lionheart W R B 2004 Geometric methods for anisotropic inverse boundary value problems In Bingham K, Kurylev Y V and Somersalo E (Eds.) New Analytic and Geometric Methods in Inverse Problems Springer 237-252

Lozano A, Rosell J and Pallás-Areny R 1995 Errors in prolonged electrical impedance measurements due to electrode repositioning and postural changes Physiol. Meas. 16 121-130

Olsen P A and Gopinath A R 2004 Modeling inverse covariance matrices by basis expansion IEEE Trans. Speech Audio Processing 12 37-46

Polydorides N P and Lionheart W R B 2002 A Matlab tool-kit for three dimensional electrical impedance tomography: a contribution to the EIDORS project Meas. Sci. Technol. 13 1871-1883

Soleimani M, Gómez-Laberge C and Adler A 2006 Imaging of conductivity changes and electrode movement in EIT Physiol. Meas. 27 S103-S113 